\title{
The use of del Nido cardioplegia for multiple cardiac surgery in adults
}

\author{
Wan Kee Kim, Joon Bum Kim \\ Department of Thoracic and Cardiovascular Surgery, Asan Medical Center, University of Ulsan College of Medicine, Seoul, Korea \\ Correspondence to: Joon Bum Kim, MD, PhD. Department of Thoracic and Cardiovascular Surgery, Asan Medical Center, University of Ulsan College \\ of Medicine, 88, Olympic-ro 43-gil, Songpa-gu, Seoul 05505, Korea. Email: jbkim1975@amc.seoul.kr. \\ Comment on: Ad N, Holmes SD, Massimiano PS, et al. The use of del Nido cardioplegia in adult cardiac surgery: A prospective randomized trial. J \\ Thorac Cardiovasc Surg 2018;155:1011-8.
}

Submitted Aug 15, 2018. Accepted for publication Aug 22, 2018.

doi: $10.21037 /$ jtd.2018.08.130

View this article at: http://dx.doi.org/10.21037/jtd.2018.08.130

Since Melrose et al. introduced potassium chloride induced cardiac arrest and calcium chloride induced recovery of heart beat (1), unceasing efforts have been made to improve the efficacy of myocardial protection during ischemic arrest for cardiac surgery. Buckberg proposed multi-dose perfusion of cold blood cardioplegia with potassium in 1977 , and this technique has been generally used even in the current era. Despite the popular use of the Buckberg solution or its modifications, demands for more convenient solutions that could allow better visualization of the surgical field through a single infusion have led investigators to search for cardioplegic agents with longer durability, such as the histidine-tryptophan-ketoglutarate solution. del Nido cardioplegia is one of those solutions that provide a longer duration of cardiac arrest through a single infusion than does the Buckberg solution, and its use has expanded to pediatric and adult cardiac surgery (2).

The del Nido solution is composed of $20 \%$ blood and 80\% Plasma-Lyte A (Baxter Healthcare Corporation, Deerfield, IL, USA; containing $\mathrm{KCl}, \mathrm{NaHCO}_{3}$, mannitol, $\mathrm{MgSO}_{4}$, and lidocaine). This type of mixture prevents excessively high potassium levels in cardiomyocytes, thus blocking the sequential calcium ion influx during ischemic cardiac arrest (3). In addition, because the del Nido cardioplegia solution is a combination of substrates (blood) and buffers $\left(\mathrm{NaHCO}_{3}\right)$ as well as membrane stabilizers (lidocaine) (4), it has been considered to be potent and with similar stability in the recovery of myocardial contraction to that of blood cardioplegia. On the other hand, the histidinetryptophan-ketoglutarate solution has been suspected to lead to ventricular fibrillation after the release of the crossclamp (5).

Although many studies have shown acceptable outcomes of the use of del Nido in a single cardiac procedure in adult patients, the safety of this cardioplegia in multiple or complex cardiac procedures has not been well demonstrated. Only some observational studies have shown acceptable outcomes of the del Nido solution compared with blood cardioplegia (6). In light of this issue, Ad et al. (7) published a prospective randomized controlled trial comparing perioperative outcomes between the use of del Nido $(n=48)$ and their modified whole-blood cardioplegia formula $(\mathrm{n}=41)$ for patients undergoing coronary arterial bypass surgery with or without cardiac valve procedures. They concluded that the routine use of del Nido cardioplegia in adult patients may be safe and could enhance the surgical workflow with good clinical outcomes, based on their findings of a higher rate of recovery in spontaneous rhythm $(\mathrm{P}=0.023)$, fewer patients who required inotropic support $(\mathrm{P}=0.05)$, and better washout of intravascular troponin $(\mathrm{P}=0.053)$ in the del Nido group. In addition, the del Nido group had a shorter cross-clamp time $(\mathrm{P}=0.018)$. Their findings, however, did not reach statistical significance (alpha was set at $\mathrm{P}<0.001$ ).

Ad et al. (7) should be commended for their efforts in investigating this timely issue through a randomized trial, despite the difficulties in selecting the type of randomization for artificial chemical compounds. The merit of a randomized trial lies on "randomizing" the baseline profiles of patients, thus allowing the study to possibly yield 
the true impact of the treatment by eliminating potential confounders, compared with observational studies. In this regard, the cited study validated the findings of previous observational studies. Furthermore, lowering the cardiac ischemic time by reducing procedural interruptions is an important point in the choice of cardioplegia solution.

However, in the cited study, the collected number of patients might be insufficient to reach their set of "alpha", to allow determining the true impact of the del Nido cardioplegia solution. As the authors revealed in their "Statistical analyses" section, the study was stopped after interim evaluations showed meaningful findings beyond non-inferiority. Accordingly, an alpha level of $\mathrm{P}<0.001$ was determined as analyses were shifted to a superiority methodology. Although Ad and colleagues () could not show statistical significance, their findings are significant in that their study with only 89 patients demonstrated the potential benefit of the use of del Nido solution within the traditional alpha level $(\mathrm{P}<0.05)$. For further approval of the use of del Nido solution in complex adult cardiac surgery, more robust results based on evaluations of a larger cohort are warranted.

\section{Acknowledgements}

None.

\section{Footnote}

Conflicts of Interest: The authors have no conflicts of interest to declare.

\section{References}

1. Melrose DG, Dreyer B, Bentall HH, et al. Elective cardiac arrest. Lancet 1955;269:21-2.

2. Matte GS, del Nido PJ. History and use of del Nido cardioplegia solution at Boston Children's Hospital. J Extra Corpor Technol 2012;44:98-103.

3. Govindapillai A, Hua R, Rose R, et al. Protecting the aged heart during cardiac surgery: use of del Nido cardioplegia provides superior functional recovery in isolated hearts. J Thorac Cardiovasc Surg 2013;146:940-8.

4. Kotani Y, Tweddell J, Gruber P, et al. Current cardioplegia practice in pediatric cardiac surgery: a North American multiinstitutional survey. Ann Thorac Surg 2013;96:923-9.

5. Braathen B, Jeppsson A, Schersten H, et al. One single dose of histidine-tryptophan-ketoglutarate solution gives equally good myocardial protection in elective mitral valve surgery as repetitive cold blood cardioplegia: a prospective randomized study. J Thorac Cardiovasc Surg 2011;141:995-1001.

6. Kim WK, Kim HR, Kim JB, et al. del Nido cardioplegia in adult cardiac surgery: beyond single-valve surgery. Interact Cardiovasc Thorac Surg 2018;27:81-7.

7. Ad N, Holmes SD, Massimiano PS, et al. The use of del Nido cardioplegia in adult cardiac surgery: A prospective randomized trial. J Thorac Cardiovasc Surg 2018;155:1011-8.
Cite this article as: Kim WK, Kim JB. The use of del Nido cardioplegia for multiple cardiac surgery in adults. J Thorac Dis 2018;10(Suppl 33):S3902-S3903. doi: 10.21037/jtd.2018.08.130 